\title{
Calibratability of mid-frequency aperture arrays with self-holography
}

\author{
Cornelis R. Wilke, ${ }^{\text {a }}$ Stefan J. Wijnholds $\odot,{ }^{\text {a,b,** }}$ and Jacki Gilmore $\odot^{\text {a }}$ \\ ${ }^{a}$ University of Stellenbosch, Department of Electrical and Electronic Engineering, Stellenbosch, \\ South Africa \\ ${ }^{b}$ Netherlands Institute for Radio Astronomy, Dwingeloo, the Netherlands
}

\begin{abstract}
The stations of the mid-frequency aperture array (MFAA) envisaged for the second phase of the Square Kilometer Array (SKA) are expected to consist of order $10^{3}$ to $10^{4}$ receive paths. This will make calibration procedures based on the array covariance matrix computationally expensive. Recently, self-holography (SH) was proposed to overcome this issue. This method assumes that the signal from the chosen calibration source can be sufficiently well isolated. We study the signal-to-interference ratio (SIR) of the most suitable calibration source over a sidereal day for a representative MFAA station located on the SKA site in South Africa. We find that an SIR of $20 \mathrm{~dB}$ or higher is achievable over (most of) a sidereal day. This is well above the $11.5 \mathrm{~dB}$ needed to keep the beamformer efficiency after calibration above $99 \%$. We therefore conclude that $\mathrm{SH}$ is a promising candidate for station calibration of the MFAA stations. $\odot$ The Authors. Published by SPIE under a Creative Commons Attribution 4.0 International License. Distribution or reproduction of this work in whole or in part requires full attribution of the original publication, including its DOI. [DOI: 10.1117/1.JATIS.8.1.011008]
\end{abstract}

Keywords: calibration; self-holography; aperture arrays; Square Kilometer Array.

Paper 21075SS received Jul. 27, 2021; accepted for publication Nov. 1, 2021; published online Nov. 19, 2021.

\section{Introduction}

The Square Kilometer Array (SKA) science book ${ }^{1}$ provides a broad overview of the science enabled by the SKA. These science cases include the Billion Galaxy HI Survey, ${ }^{2}$ studies of the transient Universe, ${ }^{3}$ and the Search for Extraterrestrial Intelligence. ${ }^{4}$ For these ambitions, field of view, sensitivity, and survey speed are key design parameters. As field of view is inherently determined by the size of an individual receiving element, this has driven design studies toward large- $N$ interferometer arrays consisting of many small-diameter receivers. All-digital aperture arrays are the extreme limit of this idea and offer several attractive features.

1. The individual antennas have a low directivity and can therefore effectively provide a hemispheric field of view.

2. To mitigate the computational cost, ${ }^{5,6}$ the antennas can be grouped into subarrays or stations, with signals that are beamformed to make the station act as a single receiving element of the interferometer array.

3. As computing resources become more affordable, the compute platform can be upgraded over time, allowing the telescope system to process an increasing number of beams and thereby extending its field of view. ${ }^{7}$

However, as the size of the individual antennas scales proportional to the observed wavelength, the number of receive paths involved rises sharply with frequency. With current technology, this makes aperture arrays viable up to L-band. For this reason, aperture arrays are currently successfully operated at low observing frequencies as demonstrated by LOFAR $^{8}$ and $\mathrm{MWA}^{9}$ and by the development of the low-frequency instrument of the SKA (SKA-Low). As aperture arrays at L-band frequencies are more challenging, they are part of the SKA observatory development program toward the second phase in the life of the SKA.

*Address all correspondence to Stefan J. Wijnholds wijnholds@astron.nl 
The stations of the mid-frequency aperture array (MFAA) are envisaged to operate in the 450to $1450-\mathrm{MHz}$ range and to have order $10^{3}$ to $10^{4}$ receive paths. ${ }^{10}$ This will make standard calibration procedures based on the array covariance matrix ${ }^{11,12}$ computationally expensive. A new technique dubbed self-holography $(\mathrm{SH})$ was proposed to mitigate this issue using the correlations between the signals from the individual receive paths with the signal from a single reference beam pointed at a suitable calibration source. ${ }^{13}$ The error budget of this method has been carefully analyzed. ${ }^{14} \mathrm{SH}$ was successfully demonstrated in practice on a LOFAR station ${ }^{14}$ and an SKA-Low prototype station. ${ }^{15}$ The latter demonstration used a variation of the originally proposed method in which signals beamformed toward a grid of pointing directions were correlated with the reference beam signal. It was shown that these two approaches are, under certain conditions, equivalent. ${ }^{16}$

In this paper, we use the analysis method developed earlier ${ }^{14}$ to assess the suitability of SH for calibration of the MFAA stations using celestial continuum sources excluding the Sun over a sidereal day. The Sun is excluded as it will likely be partially resolved on some baselines within an MFAA station and exhibits variability with time. This implies that a different strategy needs to be developed for daytime calibration, e.g., using satellite signals. Development of such a strategy is considered to be outside the scope of this paper. For our analysis, we use the design of the MFAA science demonstrator dubbed mid-frequency aperture array transient and intensitymapping system (MANTIS) ${ }^{10}$ and a realistic sky model derived from the National Radio Astronomy Observatory Very Large Array Sky Survey (NVSS),${ }^{17}$ the SUMSS,${ }^{18}$ and the Haslam map. ${ }^{19}$ We demonstrate that the proposed MANTIS stations are large enough to provide sufficient spatial selectivity to isolate a suitable calibration source at any sidereal time when the stations are located on the South-African SKA site.

This paper is organized as follows. In the next section, we provide an introduction to the $\mathrm{SH}$ method and its error budget, the envisaged MANTIS system, and the required station calibration accuracy. In Sec. 3, we describe how we obtained a realistic and complete sky model for the purpose of our analysis. This model plays a key role in the simulations, described in Sec. 4, used to determine the signal-to-interference ratio (SIR) of the available calibration sources, which is a key parameter for determining the quality of the receive path gain estimates provided by SH. In the final section, we discuss the results from the simulations and present our conclusions.

\section{Preliminaries}

\subsection{Self-Holography}

The holographic measurement technique ${ }^{20}$ is commonly used to assess the surface accuracy of reflector antennas. The technique exploits the Fourier relation between the voltage response pattern of an antenna and the electric field distribution in its aperture plane. The amplitude of the voltage response pattern is measured by scanning the antenna under test over the direction of a transmitter while the phase of the response pattern is measured using a second non-scanning reference antenna. For an aperture array, the direction-independent gains of its receiving elements are the equivalent of the surface accuracy of a reflector antenna. Scanning the array under test is done by forming a grid of beams around an isolated test source. Interestingly, such a beam can also be the signal of an individual receive path in the array. Since the holographic measurement is done using the array as both antenna under test and reference antenna, the name SH was appropriately chosen. ${ }^{13}$

A key assumption in (self-)holography is that the reference antenna isolates the signal from the source used for calibration perfectly from other signals received by the system. Even if we assume that calibration is done at frequencies without radio frequency interference, suppression of contaminating, or interfering, signals from sources other than the calibration source will not be perfect in practice. This causes a bias in the SH measurements. This bias was carefully studied ${ }^{14}$ and was found to be inversely proportional to the SIR of the measurement, which is defined as

$$
\operatorname{SIR}=\sigma_{c}(P-1) \mathbf{1} \oslash\left|\sum_{i} \sigma_{i}\left(\mathbf{a}_{i}\left(\mathbf{a}_{i}^{H} \mathbf{1}\right)-\mathbf{1}\right)\right|
$$


Here $\sigma_{c}$ is the power of the calibration source, $\sigma_{i}$ is the power of the $i$ 'th interfering source, $P$ is the number of receive paths in the array, and the $P$-element vector $\mathbf{a}_{i}$ contains phasors representing the geometrical delay of the $i$ 'th interfering source for each receiving element with respect to the phase reference point of the array. The symbol $\oslash$ denotes element-wise division, and 1 denotes a vector filled with ones. This equation signifies that the SIR may vary across the array due to unfortunate array and source geometries resulting in destructive interference of the source signals on specific baselines. As an individual receiving element contributes to many baselines in large arrays, the impact of unfortunate baselines either averages out in the case of irregular arrays, as demonstrated in our previous study, ${ }^{14}$ or affects all elements in a similar way in the case of a regular array layout. This observation allows us to average all elements of the vector to obtain a single SIR value for the full array. In practice, this single SIR value provides a good quality metric as the calibration errors found in simulated observations are inversely proportional to the SIR. ${ }^{14}$

\subsection{MANTIS}

The MANTIS ${ }^{10}$ will be a science demonstrator for the SKA and is planned to be built at the SKA site in South Africa among the existing SKA-Mid and MeerKAT systems. It will serve as a reference for the science possibilities of a fully populated MFAA system, while also providing insight on cost and technology performance. The MANTIS will integrate well with the existing MeerKAT and SKA-Mid telescopes. For example, the wide field of view of the MANTIS will enable the monitoring and detection of transient events that, once detected, can be followed up by observations with the more sensitive MeerKAT and SKA-Mid telescopes.

The theoretical capabilities of aperture arrays are very attractive for radio astronomy. However, the system engineering team realized that some of these capabilities will be unreachable due cost scaling and limited available budgets. The MANTIS will therefore provide scientists with a testbed from which budget-viable technology can be tested and optimized. The lessons learned from this experiment will form an integral part of the definition and design of the full MFAA system. Calibration of the MANTIS is a crucial aspect that will have to be studied in detail to ensure that viable scaling can be done to a full MFAA system.

The exact specifications of the MANTIS are not yet finalized. However, the aim is to consider a system that closely resembles an MFAA station. Based on this and the planned science cases, the following preliminary specifications seem the most reasonable: ${ }^{10}$

- frequency range: 450 to $1450 \mathrm{MHz}$;

- collecting area: 1500 to $2500 \mathrm{~m}^{2}$;

- sensitivity: 38 to $63 \mathrm{~m}^{2} / \mathrm{K}$;

- field-of-view: $200 \mathrm{deg}^{2}$ at $1 \mathrm{GHz}$;

- bandwidth: $>500 \mathrm{MHz}$ and

- transient buffering.

These specifications provide enough information to define a realistic receiver system to assess the calibratability of such an array at a given frequency using SH. The only outstanding aspect required to conduct the study is a realistic sky model. Section 3 discusses the steps that were involved in deriving a sky model that is suitable for calculating the SIR in a computationally tractable manner while representing the true sky realistically.

\subsection{Required Station Calibration Accuracy}

The key goal of station calibration is to characterize the individual receive paths and equalize their differences so that a sufficiently high beamforming efficiency is achieved when pointing the station. When the errors are represented as a complex-valued error with relative RMS magnitude $\epsilon$ with respective to the magnitude of the complex-valued direction-independent receive path gain, the beamformer efficiency is given by ${ }^{21}$ 


$$
\eta_{\mathrm{BF}}=1-2 \epsilon^{2}
$$

This result implies that a beamformer efficiency of $99 \%$ requires $\epsilon \leq 7.1 \%$, whereas a beamformer efficiency of $98 \%$ requires $\epsilon \leq 10 \%$.

The RMS gain error introduced by ignoring the presence of interfering sources is inversely proportional to the SIR with a proportionality constant of about unity. ${ }^{14}$ To achieve the $98 \%$ beamformer efficiency, we thus need an SIR of at least $10 \mathrm{~dB}$, while for $99 \%$ beamformer efficiency, we require an SIR of $11.5 \mathrm{~dB}$ or higher.

\section{Modeling the Sky}

For our simulations, we need a sufficiently accurate model of the sky for which the SIR can be evaluated in a reasonable amount of time. The Haslam map might seem an obvious choice for the model since it covers the entire sky. However, modeling the entire sky using the Haslam map is computationally expensive for an SIR analysis for MANTIS. Point source catalogs (PSCs) are a logical alternative because they provide a comprehensive overview of the flux density distribution across the sky and are much easier to work with. However, individual PSCs do not cover the entire sky. Moreover, to maximize the spectral accuracy of the sky model, it is important that a chosen PSC has an observing frequency that is reasonably close to the operating band of the MANTIS. These requirements, together with the geographical placement of the MANTIS, introduced a unique modeling challenge.

The SUMSS source catalog compiled using the Molonglo Observatory Synthesis Telescope (MOST) at $843 \mathrm{MHz}^{18}$ is the only PSC for the southern hemisphere sky with an appropriate observing frequency. The only drawback is that its coverage is limited to declinations $\delta<-30 \mathrm{deg}$, which is only around half of the sky as seen from the geographical location of MANTIS. Furthermore, Galactic latitudes $-10 \mathrm{deg}<b<10 \mathrm{deg}$ are unobserved, which means that the Galactic plane (GP) is entirely omitted. This coverage issue can partially be solved using the NVSS at $1400 \mathrm{MHz},{ }^{17}$ which covers the entire sky north of $\delta=-40 \mathrm{deg}$. Coverage of the GP remains an issue, as can be seen from Fig. 1. However, it will be seen that PSCs do not suffice to accurately model the GP anyway and that the Haslam map is needed.

The flux and positional uncertainties of the sources in PSCs are expected to have a negligible impact on the accuracy of the SIR calculations in this analysis. It therefore seems reasonable to directly combine the PSCs by only applying an appropriate spectral scaling. Figure 2 shows a comparison of the flux of a number of common sources, before and after scaling those from the NVSS from 1400 to $843 \mathrm{MHz}$ using the spectral index (0.83) that was derived in an earlier study. ${ }^{18}$ A close agreement is observed, which confirms that the scaling is done correctly.

In addition to the fact that the SUMSS catalog does not cover the GP region, the sky cannot be accurately modeled with only PSCs due to the presence of diffuse emission, as can be clearly seen in Fig. 3. The diffuse emission will make a relatively small contribution to the visibilities of MANTIS, but it remains a source of interference when performing SH calibration and should therefore be accounted for in our analysis. The Haslam map can be used to account for the diffuse emission, but this should not result in taking into account the fluxes of point sources (PSs) twice.

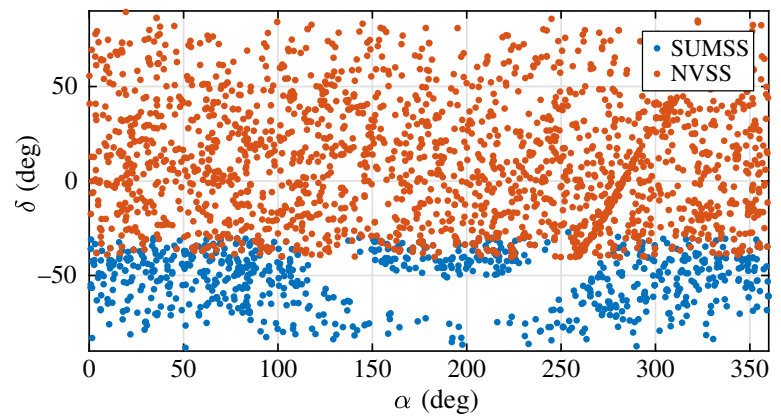

Fig. 1 PS positions from the NVSS and SUMSS catalogs for $S>1 \mathrm{Jy}$. 


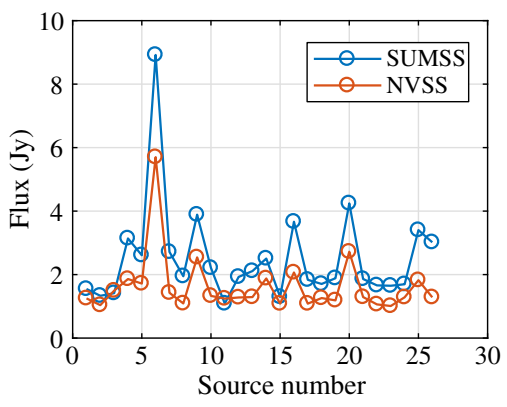

(a)

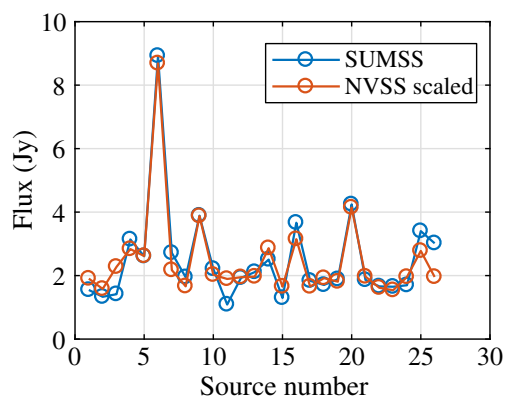

(b)

Fig. 2 Fluxes of common PSs in the NVSS and SUMSS catalogs (a) before and (b) after scaling NVSS flux from 1400 to $843 \mathrm{MHz}$ using spectral index $\alpha=0.83$.

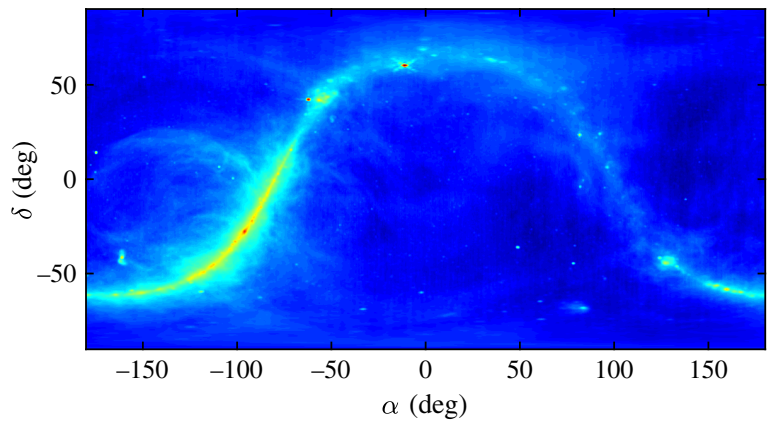

Fig. 3 Haslam 408-MHz all-sky brightness distribution atlas.

A quick inspection of Fig. 3 shows that the highest concentration of diffuse emission is located along the GP. We therefore modeled this region of the sky using the Haslam map, whereas the rest of the sky is modeled using the PSCs.

The Haslam map is a brightness map in units of Kelvin. A conversion to an intensity map (IM) with units of Jy/sr was therefore necessary before it could be combined with the PSCs. This conversion was done using ${ }^{20}$

$$
T_{\mathrm{B}}=\frac{c^{2} S}{2 k_{\mathrm{b}} f^{2}}=\frac{\lambda^{2} S}{2 k_{\mathrm{b}}} .
$$

Next, a conversion to units of Jy/pixel was done by sampling the IM at a resolution comparable to the spatial resolution of MANTIS. Finally, the flux density was scaled to $843 \mathrm{MHz}$ using a spectral index of 0.55 . If these conversions are done correctly, it is expected that there will be a good flux density comparison with the PSs from the PSCs in regions containing very little to zero diffuse emission. To test this, a collection of bright spots in the Haslam map in isolated regions (far from the GP) were chosen for declinations $\delta<-40 \mathrm{deg}$ for comparison with PSs in the SUMSS catalog. In this way, only one frequency scaling factor (Haslam from 408 to $843 \mathrm{MHz}$ ) had to be taken into account as a factor impacting the accuracy of the comparison. Due to the superior resolution of the SUMSS, each bright spot (an apparent PS) in the IM likely contains several SUMSS PSs. An effort was therefore made to only choose bright spots that are represented by one or two PSs with flux densities that are much higher than the remaining PSs in the bright sport. In this way, it could be assumed that the total flux (sum of the pixels) of the bright spot will be dominated by the flux of the strongest PSs that it contains. The uncertainty in the source parameters in both the IM and the PSC is inversely proportional to the flux density, which further supports this methodology. The analysis for one of these sources is shown in Fig. 4, in which close agreement between the total flux of the IM and SUMSS can be seen. Analysis for other bright spots showed similar results, which confirms that the flux scaling in 


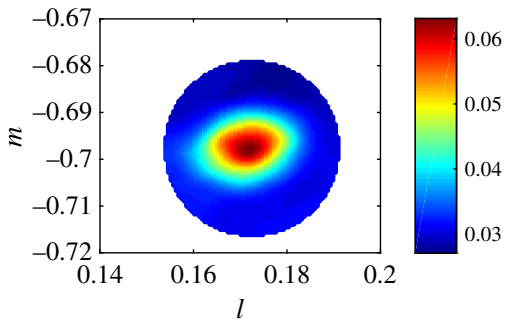

(a)

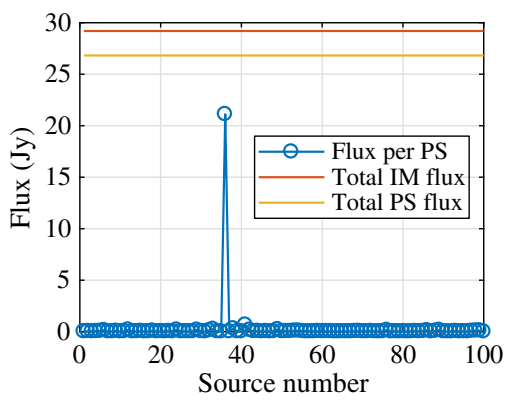

(b)

Fig. 4 Flux density comparison of a bright spot in an IM (derived from the Haslam map) with the sources in the same region as listed in the SUMSS catalog. The LST of the analysis is 23:24. (a) Bright spot in the IM used for analysis. Scale is in Jy/pixel at $843 \mathrm{MHz}$. (b) Flux per SUMSS PS in the same region. The brightest source (21 Jy) is listed as SUMSS J040848750720. A comparison of the total flux in the IM versus the total PS flux is also shown.

frequency of the IM is sufficiently accurate. The remaining differences can be attributed to underlying imperfections such as the fitting techniques used in SUMSS and the fact that each pixel in the IM contains a contribution from the flux of the rest of the sky (uncorrected for sidelobe response). A similar analysis for regions in the GP showed a very large flux difference between PSCs and the Haslam map, which was expected given the large concentration of diffuse emission in that region.

We therefore concluded that an appropriate sky model can be constructed using the Haslam map for the GP, i.e., for Galactic latitudes $-10 \mathrm{deg}<b<10 \mathrm{deg}$, and the PSCs for the remaining regions of the sky. In this way, the number of source components to be evaluated for our analysis was kept tractable while the distribution of power across the sky is still accurately represented.

\section{Simulations}

\subsection{Simulated Array}

The geographic coordinates of the array were assumed to be $30.72^{\circ} \mathrm{S}$ and $21.41^{\circ} \mathrm{E}$. A square regular array configuration was assumed with interelement spacing $d=\lambda / 2$ at $1 \mathrm{GHz}$. To ensure a collecting area of at least $1500 \mathrm{~m}^{2}$ at $1.45 \mathrm{MHz}, 336$ elements on a side of the array were required, assuming an individual antenna directivity of 4 .

The preliminary MANTIS specifications make no direct mention of subdividing the array into tiles. However, they do give a minimum field-of-view specification of 200 square deg at $1 \mathrm{GHz}$. Assuming that the elements of a tile have a regular square layout with a spacing of $\lambda / 2$ at $1 \mathrm{GHz}, 200$ square deg corresponds to a maximum of 7 elements on a side of a tile $(M=7)$. To assess the effect of tile size, we studied tile sizes of $M=1, M=2$, and $M=4$. We assume perfect beamforming at the tile level.

The embedded element patterns (EEPs) of the receiving elements were assumed to be a sinusoidal function of elevation $\theta$ such that the power attenuation in the direction of a source can be calculated $\sin ^{2}(\theta)$. As the proposed MFAA station operates in the dense regime at the low end of its operating frequency range and in the sparse regime at the high end of its operating frequency range, the directivity of the individual antennas will change significantly with frequency. Although this may affect the SIR at a given sidereal time, we note that most of the suppression of other sources is provided by the station beam,${ }^{14}$ so varying EEPs does not have a significant effect on the conclusions of our analysis.

\subsection{Calibrator Selection Strategy}

The apparent power and isolation are the most important aspects to consider when selecting a calibrator for SH. ${ }^{14}$ Intuitively, this seems to rule out calibration sources in the GP due to the 


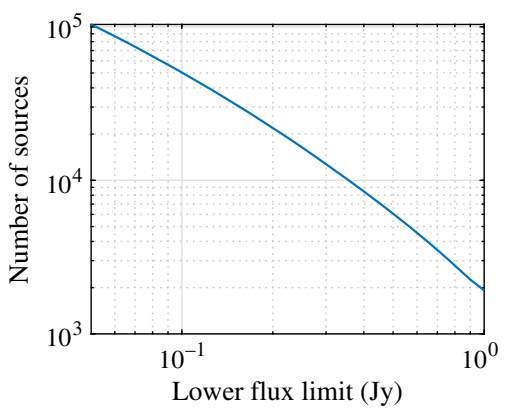

(a)

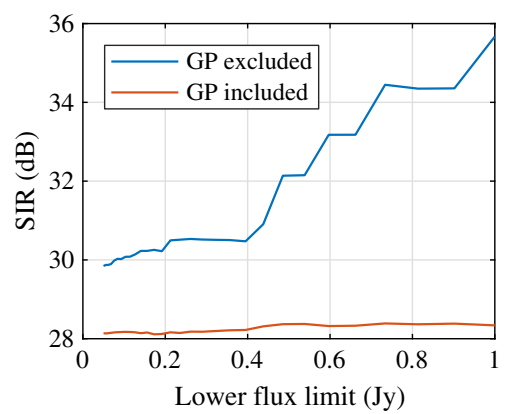

(b)

Fig. 5 (a) Cumulative source count and (b) SIR for $M=1$ versus lower flux density limit of the sources in the calibration region.

strong diffuse emission. However, our earlier analysis shows that isolation of the calibrator becomes a minor factor for sufficiently bright sources combined with a high directivity of the array. ${ }^{14}$ However, with the way we modeled the sky for MANTIS, it will be challenging to select a calibration source from the GP. We therefore limited the region from which a calibrator can be selected to regions outside the GP, where the sky was modeled using the PSCs. This region will further be referred to as the calibration region. The PS nature of the sky model in the calibration region enabled effective automatic identification and evaluation of potential calibrators.

Figure 5(a) shows the cumulative PS count versus lower flux density limit for the calibration region. The exponential nature of the number of PSs versus lower flux density limit, together with the fact that the PSs have a uniform random distribution, suggests that it is not unreasonable to assume that the brightest sources are fairly well separated at the resolution of the telescope. It was therefore assumed that the isolation of a bright source (from other bright sources) is not a concerning factor when identifying potential calibrators. A quick visual inspection confirmed this assumption. The region from which a calibrator can be selected was further limited to elevation angles $\theta>30 \mathrm{deg}$ to ensure that the required scan angle of MANTIS to reach a potential calibrator remained realistic in a practical sense.

Due to the superior resolution of the VLA and the MOST, some sources that are resolved in the NVSS and the SUMSS will be unresolved by MANTIS. Therefore, a potential calibrator in the calibration region might be a "cluster" of bright PSs that are unresolved by MANTIS. If a potential calibrator is identified, we thus need to check whether that source is part of an unresolved cluster of bright sources and determine an optimal pointing angle for this unresolved cluster. This will avoid destructive interference of signals from sources at opposite sides of the cluster on the longest baselines within the station array. A function was written that receives the positions and corresponding powers of sources stronger than $1 \mathrm{Jy}$, from which an optimal cluster (and its corresponding pointing angle) was selected based on the apparent power of its brightest component. Obviously, these clusters also contained sources with powers $<1 \mathrm{Jy}$, but these were assumed to have a negligible contribution to the integrated power of the cluster. The process of calculating the SIR from the sky model is discussed next.

\subsection{Calculating the SIR}

The SIR was calculated using Eq. (1). For a specific sidereal time, $\mathbf{a}_{i}$ is the geometric delay vector of the $i$ 'th interfering source in the calibration region or pixel in the GP region $\left(-10 \mathrm{deg}<b<10 \mathrm{deg}\right.$ ). The apparent powers $\sigma_{c}$ (calibrator) and $\sigma_{i}$ (interferer) take the attenuation of both the tile beam (for $M>1$ ) and the EEP of each antenna into account. When implementing the sum by looping over all sources in the sky, the SEFD of $0.0134 \mathrm{Jy}$ (after integration over $1 \mathrm{~s}$ and $10 \mathrm{MHz}$ ) of MANTIS is taken into account by disregarding sources that are below the SEFD.

The MANTIS sky model consists of thousands of sources to evaluate. This makes the calculation of the SIR for many instances over a sidereal day computationally expensive. At the same time, the number of sources becomes so large below a certain flux density limit that a single 
MANTIS station will reach its classical confusion limit. This effect is seen in Fig. 5, which shows the cumulative source count (at a random sidereal time) and the corresponding SIR, as a function of the lower flux density limit of the sources in the calibration region. An exponential relationship is observed in the source count while a very weak dependence is observed in the SIR for flux density limits $<400$ mJy (GP excluded). When including the GP, a very weak dependence is observed for the full lower flux density limit range (which highlights the dominance of the interference coming from the GP). A significant optimization could therefore be done by only including PSs with apparent flux densities higher than $400 \mathrm{mJy}$.

\section{Results}

The SIR was calculated using Eq. (1) at $10 \mathrm{~min}$ intervals over $24 \mathrm{~h}$ local sidereal time. The results are shown in Fig. 6. The apparent power of the calibrator $\sigma_{c}$ at each time instance is plotted on the right axis. The selection of a calibrator and its apparent power is independent of tile size and can therefore be represented by a single curve.

As expected, the SIR, in general, improves significantly as a function of increasing tile size. This is mostly due to an increase in directivity and a subsequent increase in the isolation of the calibrator. The erratic variations of the results can be explained by the movement of bright sources through the sidelobes as time passes. The discontinuities in the calibrator flux density over time indicate the instances at which the algorithm selects a new calibrator. A smooth relationship in which the attenuation of the EEP is clear is otherwise seen.

It is interesting to note that the maximum SIR does not coincide with the maximum value of $\sigma_{c}$. A closer look at the flux density distribution across the sky can explain this. Figure 7 shows the positions of all sources at the indicated times, and their corresponding apparent powers after attenuation by the EEP's and the array beam for $M=1$. Also shown is the corresponding IM (Jy/ pixel) for the GP with the pointing direction of the array beam indicated by the red cross. The arms of the cross also represent the principal planes of the array.

For $M=1$, the SIR reaches a maximum around $t=02: 00$ when $\sigma_{c}$ is around half of its maximum value over the $24 \mathrm{~h}$. Several hours later (at around $t=11: 00$ ), $\sigma_{c}$ reaches its maximum value while the SIR has decreased slightly. This is explained by the plots in the top two rows in Fig. 7, which show the corresponding sky maps at both times. In both instances, the calibrator is close to the GP, and as a result, the array beam samples the GP through its sidelobes in the principal plane with relatively low attenuation. However, at the second instance $(t=11: 02)$, when $\sigma_{c}$ reaches its maximum, the sidelobes in the principal planes of the array cut through much brighter regions of the GP than in the first instance $(t=02: 12)$, resulting in a much higher level of interference.

A relatively sharp drop in the SIR is seen around $t=17: 00$ followed by a sharp rise a short time later. Initially, the SIR decreases as $\sigma_{c}$ goes down (for $t>11: 00$ ), which is an expected trend. However, a few moments later, the SIR increases significantly while $\sigma_{c}$ remains relatively

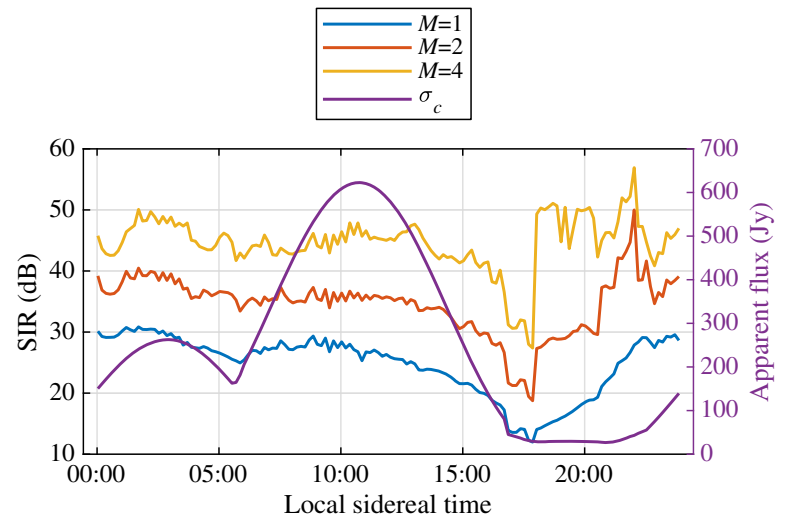

Fig. 6 The SIR at 10 min intervals over $24 \mathrm{~h}$ local sidereal time. The apparent power of the calibrator is shown on the right axis. 

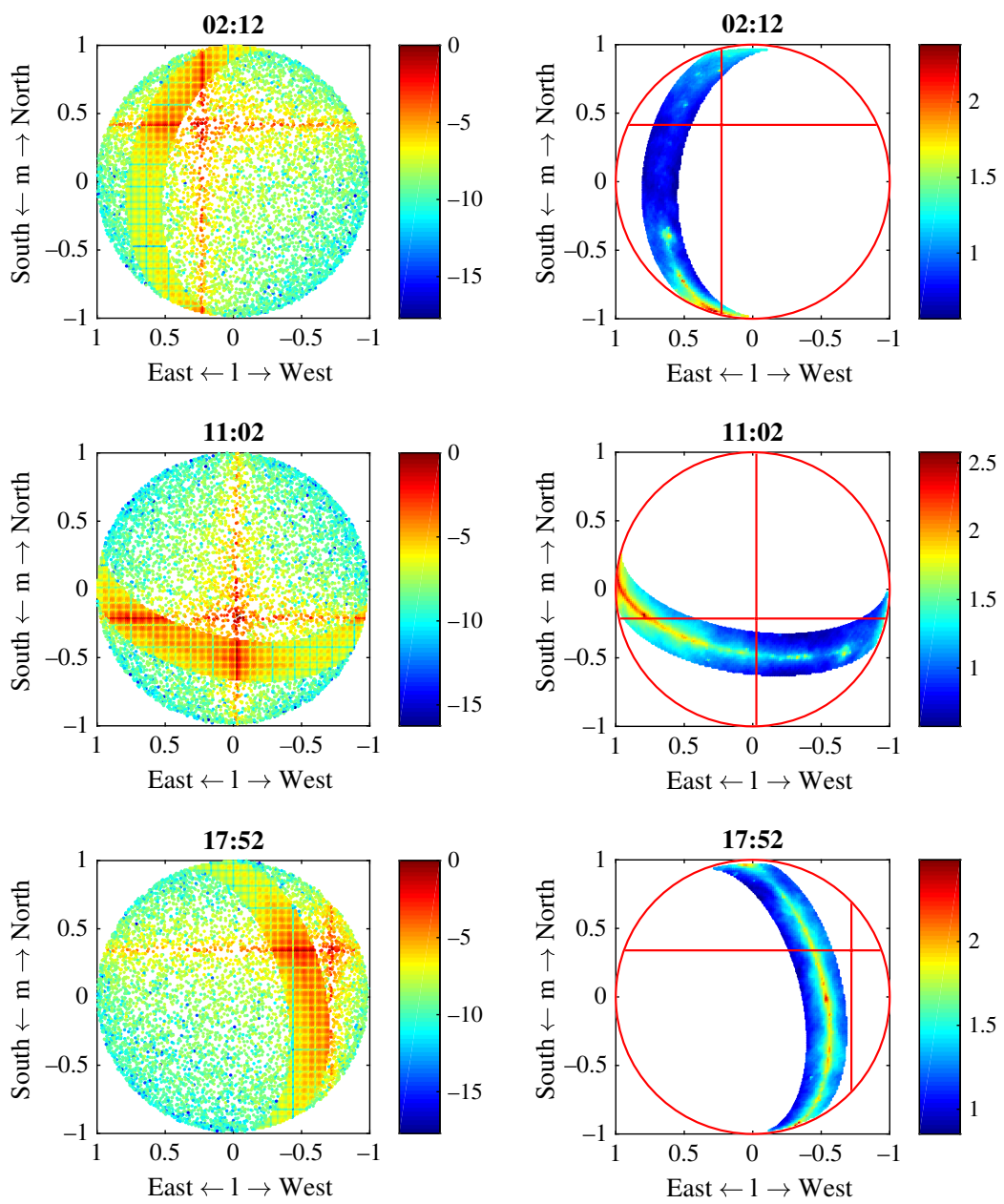

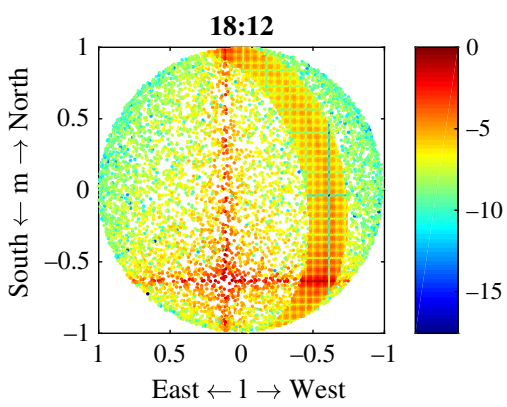

(a)

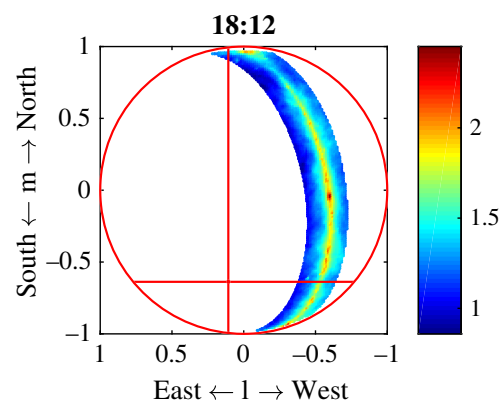

(b)

Fig. 7 (a) Positions of sources at the indicated local sidereal times. The logarithmic color scale indicates their apparent powers after attenuation by EEPs and the array beam. The powers are normalized to the brightest source. (b) The corresponding IM (Jy/pixel) for the GP with the pointing direction of the array beam is indicated by the red cross. The arms of the cross also represent the principal planes of the array.

constant. At $t=17: 52$, the array beam is pointed at a calibrator close to the horizon with the GP and its centre, where the spatial concentration of power is the highest, close by to the left of the pointing direction. It is visibly clear that the array beam is sampling a significant portion of the GP through its sidelobes with relatively low attenuation due to its close proximity to the main beam. A few moments later at 18:12, the array beam shifts to a new calibrator with similar apparent power but located further away from the GP. The increased distance between this calibrator and the GP results in higher attenuation (by the array beam) of the power coming from it. This results in a sudden increase in the SIR. As expected, the exact increase in SIR at this point 
strongly depends on tile size. For a fixed calibration scenario, an increase in tile size will always result in a higher SIR due to the higher directivity imposed by the tile beams. After switching to the new calibrator, the SIR continues to increase as a function of time while the apparent power of the calibrator remains relatively constant. This is because the calibrator moves along an equalsensitivity contour of the EEPs while the GP moves closer to the horizon, resulting in an increase in its attenuation by the EEPs. Eventually, the entire GP dips partially below the horizon, causing the SIR to spike for $M=2$ and $M=4$. This spike is much lower for $M=1$ due to its much lower attenuation at the horizon.

\section{Discussion and Conclusions}

In this paper, we analyzed the calibratability of an MFAA station when using SH on astronomical continuum sources excluding the Sun. To do this, a realistic sky model was simulated for an array that is based on the preliminary specifications of the MANTIS.

The SIR was analyzed over a full sidereal day for a station placed at the SKA site in South Africa. The results revealed that the isolation and position of a potential calibrator close to the GP has a larger impact on the SIR than we originally anticipated. For example, the brightest potential calibrator might be located such that a principal plane of the array cuts through a bright region of the GP, resulting in a lower SIR than achievable with a fainter source that is more isolated from the GP. The results showed that SIRs higher than $20 \mathrm{~dB}$ are achievable across a full sidereal day, which is well above the $11.5 \mathrm{~dB}$ required to achieve $99 \%$ beamformer efficiency as explained in Sec. 2.3. The suboptimal calibrator selection strategy implies that these results give a minimum value for the SIR achievable with MANTIS. It can therefore be concluded with confidence that SH will be able to produce high-quality calibration results when applied to the MANTIS. This further implies that a system like an MFAA station can benefit from the significantly lower computing requirements of SH compared with full covariance matrix-based calibration methods.

\section{Acknowledgments}

This work was supported by the National Research Foundation (NRF) of South Africa, the South African Radio Astronomy Observatory (SARAO), and the Netherlands Organisation for Scientific Research. The authors declare that they have neither a financial interest in the material presented in this manuscript nor other potential conflicts of interest in having this material published.

\section{Data, Materials, and Code Availability}

The code used to generate the results in this paper can be publicly found at https://github.com/ wilkecornelis/SH-for-MFAA-code.git.

\section{References}

1. T. L. Bourke et al., Advancing Astrophysics with the Square Kilometre Array, Dolman Scott Ltd. for SKA Organization, Manchester (2015).

2. S. L. Blyth et al., "Exploring neutral hydrogen and galaxy evolution with the SKA," in Proc. Sci., Vol. 218 (2014).

3. R. Fender et al., "Transient astrophysics with the Square Kilometre Array," in Proc. Sci., Vol. 218 (2014).

4. A. P. V. Siemion et al., "Searching for extraterrestrial intelligence with the Square Kilometre Array," in Proc. Sci., Vol. 218 (2014).

5. S. J. Wijnholds and R. Jongerius, "Computing cost of sensitivity and survey speed for aperture array and phased array feed systems," in IEEE Africon (2013).

6. R. Jongerius et al., "An end-to-end computing model for the Square Kilometre Array," IEEE Comput. 47, 48-54 (2014). 
7. J. D. Bregman, "Cost effective frequency ranges for multi-beam dishes, cylinders, aperture arrays and hybrids," Exp. Astron. 17, 407-416 (2004).

8. M. P. van Haarlem et al., "LOFAR: the low frequency array," Astron. Astrophys. 556, A2 (2013).

9. R. B. Wayth et al., "The phase II Murchison widefield array: design overview," Publ. Astron. Soc. Aust. 35, 1-9 (2018).

10. W. A. van Cappellen et al., "MANTIS: the mid-frequency aperture array transient and intensity-mapping system," arXiv:1612.07917, MANTIS white paper (2016).

11. S. J. Wijnholds et al., "Calibration challenges for future radio telescopes," IEEE Signal Process Mag. 27, 30-42 (2010).

12. S. Salvini and S. J. Wijnholds, "Fast gain calibration in radio astronomy using alternating direction implicit methods: analysis and applications," Astron. Astrophys. 571, A97 (2014).

13. S. J. Wijnholds, "Calibration of mid-frequency aperture array stations using selfholography," in Int. Conf. Electromagn. Adv. Appl. (2017).

14. C. R. Wilke, S. J. Wijnholds, and J. Gilmore, "Calibratability of aperture arrays using self-holography," IEEE Trans. Antennas Propag. 69, 4527-4537 (2021).

15. U. Kiefner et al., "Holographic calibration of phased array telescopes," Radio Sci. 56, e2020RS007171 (2021).

16. S. J. Wijnholds, "Generalised self-holography," in XXXIV General Assembly and Sci. Symp. Int. Union of Radio Sci. (2021).

17. J. J. Condon et al., "NRAO VLA Sky Survey (NVSS)," Astron. J. 115(5), 1693-1716 (1998).

18. T. Mauch et al., "SUMSS: a wide-field radio imaging survey of the southern sky," Mon. Not. R. Astron. Soc. 342, 1117 (2003).

19. C. Haslam et al., "A $408 \mathrm{MHz}$ all-sky continuum survey II—the Atlas of contour maps," Astron. Astrophys. Suppl. Ser. 47, 1-143 (1982).

20. A. R. Thompson, J. M. Moran, and G. W. Swenson, Interferometry and Synthesis in Radio Astronomy, Astronomy and Astrophysics Library, Springer International Publishing, Cham, Switzerland (2017).

21. S. J. Wijnholds, "Embedded element patterns in hierarchical calibration of large distributed arrays," in General Assembly and Sci. Symp. Int. Union Radio Sci. (2020).

Cornelis R. Wilke received his BEng and MEng (cum laude) degrees and his $\mathrm{PhD}$ in electrical and electronic engineering from the University of Stellenbosch, Stellenbosch, South Africa, in 2015, 2017, and 2021, respectively. His research interests are in the design of phased array systems and array signal processing.

Stefan J. Wijnholds received his MSc degree in astronomy and his MEng degree in applied physics (both cum laude) from the University of Groningen, Groningen, the Netherlands, in 2003 and his PhD (cum laude) from Delft University of Technology, Delft, the Netherlands, in 2010. After his graduation in 2003, he joined the R\&D Department of ASTRON, the Netherlands Institute for Radio Astronomy, Dwingeloo, the Netherlands, where he works on the development of the next generation of radio telescopes based on phased array technology. From 2006 to 2010, he was also affiliated with the Delft University of Technology, Delft, the Netherlands. Since 2016, he has been affiliated with the Electrical and Electronic Engineering Department, University of Stellenbosch, Stellenbosch, South Africa, as an extraordinary (associate) professor. In 2018, he became a senior researcher at ASTRON overseeing work on the institutional technology development roadmap to address the big data challenges posed by large phased array systems. His research interests lie in the area of array signal processing, specifically calibration and imaging, and system design of the next generation of radio telescopes.

Jacki Gilmore received her BEng and MScEng (cum laude) degrees and her PhD in electrical and electronic engineering from the University of Stellenbosch, Stellenbosch, South Africa, in 2007, 2010, and 2016, respectively. She is currently a lecturer at Stellenbosch University. Her research interests include antenna and phased array system design and array signal processing. 